\title{
Latent Trajectories and Profiles of Commercial Cigarette Smoking Frequency From Adolescence to Young Adulthood Among North American Indigenous People
}

\author{
Dane Hautala, PhD ${ }^{1,}$, Kelley Sittner, PhD $^{2}$, Melissa Walls, PhD $^{3}$
}

'Department of Psychiatry, University of Minnesota Medical School, Duluth, MN; ${ }^{2}$ Department of Sociology, Oklahoma State University, Stillwater, OK; ${ }^{3}$ Department of International Health, Johns Hopkins Center for American Indian Health and Johns Hopkins Bloomberg School of Public Health, Duluth, MN

Corresponding Author: Dane Hautala, PhD, Department of Psychiatry, University of Minnesota Medical School, 1915 South Street, Duluth, MN 55812, USA. Telephone: 218-724-1665; E-mail: haut0034@d.umn.edu

\begin{abstract}
Introduction: North American Indigenous people (ie, American Indian/Alaska Native and Canadian First Nations) have the highest rates of commercial cigarette smoking, yet little is known about long-term trajectories of use among this population. The purpose of this study is to examine heterogeneous trajectories and profiles of Indigenous cigarette use frequency from early adolescence (mean age: 11.1 years) to young adulthood (mean age: 26.3 years).

Aims and Methods: Data come from a nine-wave prospective longitudinal study spanning early adolescence through young adulthood among Indigenous people in the Upper Midwest of the United States and Canada $(N=706)$. Smoking frequency was examined at each wave, and latent class growth analysis was used to examine heterogeneous patterns. Early adolescent and young adult demographics and smoking-related characteristics were examined across these latent trajectory groups.

Results: In young adulthood, $52 \%$ of participants smoked daily/near-daily, and an additional $10 \%$ smoked weekly or monthly. Four latent trajectory groups emerged: low/non-smokers $(35.2 \%)$ who had low probabilities of smoking across the study; occasional smokers $(17.2 \%)$ who had moderate probabilities of smoking throughout adolescence and declining probabilities of smoking into young adulthood; mid-adolescent onset smokers $(21.6 \%)$ who showed patterns of smoking onset around mid-adolescence and escalated to daily use in young adulthood; and early-adolescent onset smokers (25.9\%) who showed patterns of onset in early adolescence and escalated to stable daily use by late adolescence.

Conclusions: The findings suggest multiple critical periods of smoking risk, as well as a general profile of diverse smoking frequency patterns, which can inform targeted intervention and treatment programming.

Implications: Nearly two-thirds (62\%) of this sample of Indigenous people were current smokers by early adulthood (mean age $=26.3$ years), which is substantially higher than national rates in the United States and Canada. Moreover, in all but one trajectory group, smoking prevalence consistently increased over time, suggesting these rates may continue to rise into adulthood. The longitudinal mixture modeling approach used in this study shows that smoking patterns are heterogeneous, and implications for public health policy likely vary across these diverse patterns
\end{abstract}




\section{characterized by timing of onset of use, escalation in frequency of use, and stability/change over}

\section{time.}

Commercial tobacco use is the most preventable cause of mortality and chronic disease and disproportionately affects North American Indigenous people (ie, American Indian and Canadian First Nations). ${ }^{1}$ Despite heterogeneity across tribal groups, the prevalence of smoking among Indigenous youth in a nationally representative U.S. sample was over twice that of the overall sample. ${ }^{2}$ The national smoking rate among adults in the United States and Canada is approximately $14 \%-15 \%$, while the adult smoking rate for Indigenous people in the United States and Canada is $24 \%$ and $40.3 \%$, respectively. ${ }^{3,4}$ Additional evidence from the National Epidemiologic Survey on Alcohol and Related Conditions indicates that the rates of lifetime and past-year nicotine dependence are nearly 1.5 times higher among Indigenous adults compared to non-Hispanic whites. ${ }^{5}$ Prior research also shows elevated cotinine levels among Indigenous youth who do not themselves smoke, which is strongly indicative of secondhand smoke exposure. ${ }^{6}$ These high rates of direct and indirect cigarette smoke exposure have wide-ranging public health consequences for Indigenous communities.

While public health campaigns to reduce smoking have been successful nationally, Indigenous communities have been left behind. Surveillance data show slight increases in smoking prevalence among Indigenous young adults ${ }^{7}$ and an earlier age of onset of smoking among the youngest cohorts of Indigenous youth compared to older cohorts. ${ }^{8,9}$ In addition, Indigenous people have lower smoking quit ratios across the life course compared to other groups, ${ }^{10}$ and smoking is a key driver of the leading causes of death (eg, cardiovascular disease, lung cancer). Designing and implementing effective public health policies to combat these disparities require a more nuanced epidemiological profile of cigarette smoking, particularly across periods of developmental risk (ie, adolescence and early adulthood). Few prospective longitudinal studies, however, examine smoking patterns among Indigenous people and those that do tend to focus on adolescence. Prior Indigenous research shows that the onset of cigarette smoking rapidly increases from age 10 to 14 , and the cumulative probability of smoking a cigarette by 16 years of age is more than $80 \%{ }^{11}$ Daily smoking increases across adolescence and the probability of becoming a daily smoker surpasses $50 \%$ by late adolescence. $^{12}$

Smoking patterns during the early life course are heterogeneous and not well captured using standard growth-modeling techniques. Longitudinal mixture modeling approaches among non-Indigenous samples spanning adolescence through young adulthood show distinct groups of smokers, which can be differentiated by timing of onset of use, escalation in frequency/quantity of use, and stability/ change over time. ${ }^{13-20}$ Earlier age of onset of smoking increases the odds of heavy use and nicotine dependence in adolescence ${ }^{16,18,20,21}$ and decreases the odds of cessation in adulthood. ${ }^{22}$ Although daily smoking patterns primarily emerge in adolescence, young adult onset smoking patterns have been found. ${ }^{14,18,21}$ Moreover, occasional, intermittent, or light smoking patterns in adolescence are less stable over time, and either escalate to regular use or taper off by young adulthood, ${ }^{19,23}$ and represent a key group that may be responsive to targeted intervention programming. To characterize such trajectories across diverse samples, longitudinal data spanning adolescence and early adulthood is necessary.

These heterogeneous smoking patterns also vary across general demographic characteristics. Prior trajectory research shows clear socioeconomic status (SES) differences, such that low SES is associated with early-onset smoking patterns, and decreased likelihood of cessation..$^{24}$ In addition, smoking trajectory groups characterized by early-onset use and rapid escalation to heavy, daily use by mid- to late adolescence have lower GPAs in high school, ${ }^{13}$ lower overall educational attainment, and lower average income in adulthood compared to never-smokers. ${ }^{15}$ Research among Indigenous youth suggests that females start smoking at earlier ages ${ }^{12}$ and have higher odds of lifetime nicotine dependence compared to males. ${ }^{25}$ Prior longitudinal mixture modeling research also suggests that despite the earlier onset of smoking, females are more likely to mature out of smoking during the transition into young adulthood..$^{23}$ At the family level, adolescents who grow up with a caretaker who smokes have a higher risk of being an early-onset smoker with persistent patterns of use. ${ }^{14-16,18}$

Developmental smoking research using longitudinal mixture modeling demonstrates that profiles of these smoking trajectories are distinct and likely have varying implications for public health practice. Only one study of Indigenous youth examines smoking patterns within a longitudinal mixture-modeling framework. Whitesell et al. ${ }^{26}$ identified three latent smoking trajectory groups across 2 years of data among young Indigenous people (grades 6-7) in the Northern Plains. Because the data only examined early adolescence, no groups showed stable patterns of daily smoking, and there were no opportunities to observe meaningful changes in use. We extend this body of research by using prospective cigarette smoking frequency data among a within-culture sample of Indigenous people to identify heterogeneous latent trajectories from early adolescence to young adulthood, which can aid in identifying critical periods of risk for shaping public health policy. In addition, we examine smokingrelated and demographic characteristics of these smoking frequency patterns, to generate a basic demographic profile of who is likely to fit different smoking risk patterns, which can aid in developing targeted intervention and treatment programming.

\section{Methods}

\section{Sample}

Data come from an ongoing community-based participatory research project with four reservations in the Upper Midwest of the United States and four Canadian First Nations reserves. As part of confidentiality agreements at each site, the names of the communities and cultural groups are not identified. The first phase of the study consisted of eight waves of data collected annually with a target adolescent and at least one primary caregiver beginning in 2002 and ending in 2010. At baseline, eligible adolescents were aged 10-12 years, enrolled tribal members, and living on or within 50 miles of the reservation/reserve. Participants were selected from tribal enrollment records at each reservation/reserve. The study team attempted to contact all participants within this age range. The recruitment rate across all sites was $79.4 \%$. Additional details on this first phase of the study can be found in previously published reports. ${ }^{27}$ The second phase of the study followed up with participants in young adulthood, and Wave 9 data were collected in 2017-2018 (mean age: 26.3 years). In both phases, data were collected via computer-assisted and paper-and-pencil personal interviews by trained tribal community interviewers. Participants received at each measurement occasion a culturally meaningful gift and a $\$ 40$ and \$50 USD incentive during the first and second phases, respectively. The sample for the current study consisted of 735 adolescents 
who enrolled at baseline. Twenty-seven adolescents passed away by Wave 9, and two participants had no smoking-related data and were removed from the data for a final analytic sample of 706 . After accounting for deceased participants, retention rates across waves ranged from $96.2 \%$ to $64.3 \%$ (see Supplementary Appendix A for retention rates at each wave). All study protocols were collaboratively developed and implemented by community and university team members and approved by the University of Nebraska and the University of Minnesota Institutional Review Boards, and each respective community's advisory board.

\section{Measures}

\section{Smoking Frequency}

Cigarette smoking frequency was examined at each wave of the study and was used as the observed indicators in a latent class growth analysis (LCGA) model. At each wave, cigarette smoking frequency was measured by asking participants whether they had ever smoked a cigarette in their lifetime and, if so, how often in the past 12 months. For those reporting any smoking, response options in the paper-and-pencil waves (Waves 1, 2, 3, 5, 7, and 9) were none, one to two times a month, once a month, every week, nearly every day, and every day. In the three diagnostic waves (Waves 4, 6, and 8 ), response options were none, daily or near-daily, 3-4 days per week, 1-2 days per week, and less than that. Because of heavy skew on both ends of the distribution (ie, non-smoking and daily smoking), and to make response options consistent across waves, smoking frequency responses were collapsed into (0) no smoking, (1) weekly or less smoking, (2) daily or near-daily smoking categories (see Supplementary Appendix A for the breakdown of monthly and weekly smoking prevalence). This coding approach has also been used in prior trajectory studies. ${ }^{15}$

\section{Smoking-Related Characteristics}

Four smoking-related characteristics were examined. First, cumulative adolescent nicotine dependence was assessed at Wave 8 of the study using standardized scoring algorithms from the Diagnostic Interview Schedule for Children, ${ }^{28}$ which was based on DSM-IV-TR criteria $(0=$ did not meet lifetime criteria, $1=$ met lifetime criteria). Second, at Waves 5 and 7 of the study, caretakers of the participants were asked if they currently smoked $(0=$ non-smokers, $1=$ smokers $)$. Third, at Wave 9, self-reported smokers were asked whether they ever tried to quit smoking, and if so, how many quit attempts they made. Fourth, self-reported smokers were asked how many cigarettes they smoked per day on average. Counts of the number of quit attempts and cigarettes smoked per day were created from these responses.

\section{Demographics}

Four demographic characteristics at baseline were assessed, including sex $(0$ = male; 1 = female $)$, caretaker highest level of education $(0=$ less than high school, $1=$ high school diploma or GED, 2 = some college or vocational/tech school, 3 = college degree), per capita family income (income from all sources, divided by number of people in the household, divided by 1000), and residing on versus off reservation/reserve land. Four demographic characteristics at Wave 9 were assessed, including residing on versus off reservation land $(0=$ off reservation, $1=$ on reservation), participant education $(0=$ less than high school, 1 = high school diploma or GED, $2=$ some college or vocational/tech school, 3 = college degree), participant individual income (personal income divided by 1000), and participant parental status $(0=$ no children, $1=$ any children $)$.

\section{Analytic Strategy}

We used LCGA to examine trajectories of cigarette smoking frequency from Waves 1 to 9 in Mplus version 8.1. ${ }^{29}$ Because the first eight waves of data followed participants in approximately 1-year intervals, and Wave 9 did not continue with this ordering, the metric of time was expressed as a year of the study (ie, linear growth factor loadings set to $0,0.1,0.2,0.3,0.4,0.5,0.6,0.7$, and 1.4 for Waves $1-9$, respectively, to account for spacing between the waves). After identifying the best fitting unconditional latent growth curve model, 2-6 classes were examined and compared across various model fit indices and substantive interpretability. The Akaike's information criterion, Bayesian information criteria (BIC), sample-adjusted BIC, Lo-Mendell-Rubin likelihood ratio test (LMR-LRT), ${ }^{30}$ and bootstrapped likelihood ratio test (BLRT) ${ }^{31}$ were compared across successive models. Lower information criteria values and a significant likelihood ratio test indicate better model fit. To adjust for potential differences in age at the start of the study, we repeated this process and included age at baseline as a predictor of growth parameters (eg, intercept, slope) and the latent trajectory class variable.

Full-information maximum likelihood estimation was used to account for missing data in the LCGA, and participants were classified in the trajectory group in which they had the highest posterior probability of being in. Because of the inconsistent time ordering of several variables, we opted to conceptualize the demographic and smoking-related characteristics as general profile variables, rather than as formal predictors and outcomes. To account for missing data on these variables, multiple imputation by chained equations was used in Stata version $15^{32}$ to generate 50 imputed datasets, ${ }^{33}$ which included the latent trajectory class variable and predictors of attrition (ie, gender, residing on/off reservation/reserve, study location) as auxiliary variables. For a number of quit attempts and cigarettes smoked per day, imputed values were estimated for current smokers only. The profile analysis results represent the parameter estimates combined across the 50 datasets.

Because of the use of multiple imputation, standard analysis of variance and chi-square testing typically used in latent class studies are not readily available. Instead, for ease of interpretation, we report the imputed means and proportions of each profile variable for the total sample and for each latent trajectory class. For each of the profile variables, except for the number of quit attempts and average number of cigarettes smoked per day, multinomial logistic regression was used to determine statistically significant differences across the latent trajectory classes by changing the latent smoking trajectory class reference group and assessing all possible contrasts (see Supplementary Appendix B for multinomial logistic regression coefficients and $95 \%$ confidence intervals). For a number of quit attempts and average number of cigarettes smoked per day, we were interested in differences only among those who are current smokers at the last wave of assessment. To assess this, we regressed both variables on the latent smoking trajectory class, current smoking status, and an interaction between the two. We assessed the difference across classes by switching the reference category for the latent smoking trajectory variable and presenting all possible contrasts. The regression coefficient for current smoking represents the averages for these outcomes in the latent smoking trajectory class reference group, and the interaction effects represent deviations and statistical significance from this average for the included trajectory groups (see Supplementary Appendix C for regression models and 95\% confidence intervals). 


\section{Results}

\section{Frequency of Smoking Across Time}

Figure 1 shows the frequency of cigarette smoking at each wave (see Supplementary Appendix A for smoking prevalence by wave). Smoking prevalence increased consistently over time and grew from $11 \%$ at Wave 1 (mean age: 11.1 years) to $62 \%$ of the sample smoking at Wave 9 (mean age: 26.3 years). Growth was primarily driven by increases in daily or near-daily smoking from $2 \%$ at Wave 1 to $52 \%$ at Wave 9 . Weekly or less smoking rates increased slightly from early to mid-adolescence (Waves 1-6), declining thereafter. The prevalence of weekly or less smoking did not exceed $20 \%$ at any given wave, indicating that participants who smoked overwhelmingly tended to be daily or nearly daily smokers.

\section{Latent Class Growth Analysis}

Unconditional latent growth curve models were estimated to identify the best fitting model. Intercept, linear, and quadratic growth were considered, and the quadratic growth model fit the data best. Two to six latent trajectory classes were examined and compared across fit indices and substantive interpretability. The information criteria indices declined consistently across models, with the differences between models decreasing in magnitude after three classes. Starting with a six-class model, small non-interpretable groups $(<4 \%)$ emerged, so four- and five-class models were further evaluated. The LMR-LRT test comparing a five-class solution to a four-class solution was not significant; however, the BLRT was significant. Both a four- and five-class solution had early- and mid-adolescent onset groups, characterized by the onset of use in early- or mid-adolescence and rapid escalation to daily or near-daily use by late adolescence. The five-class solution had an additional class characterized by lateadolescent onset smoking, characterized by the onset of use in late adolescence and rapid escalation to daily or near-daily use by early adulthood. Because these are all variations of a common pattern, we opted for the more parsimonious four-class solution with distinct early- and mid-adolescent onset classes (Table 1).

We ran each model with age at baseline as a predictor of the growth parameters and the latent class membership. The fit indices mirrored the unconditional LCGA models and suggested a four- or five-class model best fit the data. Across all models, age at baseline was positively associated with the intercept growth factor and class membership, which indicates that the observed smoking frequency variables are incorrectly related to the latent trajectory class in the unconditional model. ${ }^{34}$ As such, age at baseline was used to estimate the class size and categorize participants in the class they have the highest posterior probability of being in..$^{35}$ The average posterior probabilities for each class ranged from .77 to .93 , and the entropy value of .75 indicated acceptable classification accuracy and class separation. ${ }^{35}$

Figure 2 shows the probabilities of smoking frequency categories across the four classes at each wave (see Supplementary Appendix A for class probabilities by wave). The largest single class, low/ non-smokers, representing one-third of the sample $(35.2 \%)$, was characterized by high probabilities (>.90) of non-smoking and low probabilities of weekly or less smoking in adolescence (Waves 1-8). This group also showed a small increase in weekly or less smoking in young adulthood (Wave 9). The next largest group, occasional smokers $(17.2 \%)$, had modest probabilities of weekly or less smoking and daily or near-daily smoking throughout adolescence (Waves 1-8) and into early adulthood and was the only class to meaningfully decline in daily or near-daily use in young adulthood (Wave 9). Mid-adolescent onset smokers (21.6\%) showed onset of weekly or less and daily or near-daily smoking around Wave 3 (mean age: 13.1 years) and steadily escalated to high probabilities (>.80) of daily or near-daily smoking by Wave 9 (mean age: 26.3 ). Early-adolescent onset smokers $(25.9 \%)$ had modest probabilities of smoking at Wave 1 (mean age: 11.1 years), but rapidly escalated to and plateaued at daily use by Wave 6 (mean age: 16.2 years).

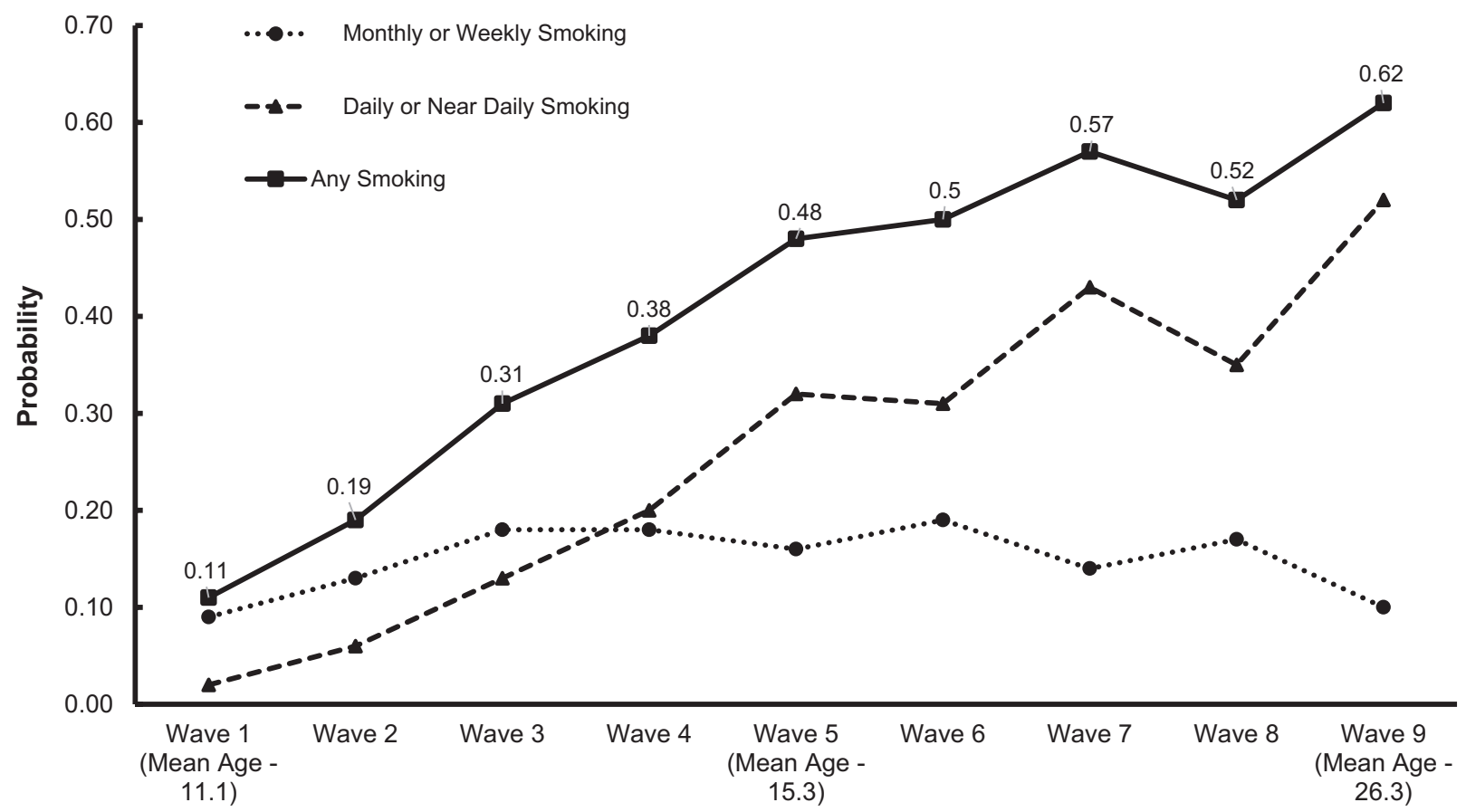

Figure 1. Growth in smoking frequency across time among the total sample $(N=706)$ 
Table 1. Latent Class Growth Analysis Model Fit Information ( $N=706)$

\begin{tabular}{|c|c|c|c|c|c|c|}
\hline & AIC & $\mathrm{BIC}$ & $\mathrm{ABIC}$ & LMR-LRT & BLRT & Entropy \\
\hline \multicolumn{7}{|c|}{ Unconditional model } \\
\hline 1 class & 9348.80 & 9367.05 & 9354.35 & & & \\
\hline 2 class & 7817.64 & 7854.14 & 7828.74 & $1482.68 *$ & $1539.16^{*}$ & .85 \\
\hline 3 class & 7562.75 & 7617.50 & 7579.40 & $253.42 *$ & $262.89 *$ & .78 \\
\hline 4 class & 7489.81 & 7562.81 & 7512.00 & $77.97^{*}$ & $80.94 *$ & .77 \\
\hline 5 class & 7446.18 & 7537.43 & 7473.93 & 49.73 & $51.63 \%$ & .74 \\
\hline 6 class & 7413.73 & 7523.23 & 7447.03 & $38.97^{*}$ & $40.45 *$ & .75 \\
\hline \multicolumn{7}{|c|}{ Conditional model with age as covariate } \\
\hline 1 class & 9166.57 & 9198.49 & 9176.26 & & & \\
\hline 2 class & 7683.22 & 7737.94 & 7699.84 & $1449.16 *$ & $1493.35^{*}$ & .85 \\
\hline 3 class & 7465.45 & 7542.96 & 7488.98 & $221.04 *$ & $227.78 *$ & .77 \\
\hline 4 class & 7393.32 & 7493.63 & 7423.78 & $79.70 *$ & $82.13 *$ & .75 \\
\hline 5 class & 7353.75 & 7476.86 & 7391.13 & 48.10 & $49.57 \%$ & .72 \\
\hline 6 class & 7330.36 & 7476.26 & 7374.66 & 32.41 & $33.40 *$ & .74 \\
\hline
\end{tabular}

$\mathrm{AIC}=$ Akaike's information criterion; $\mathrm{BIC}=$ Bayesian information criteria; $\mathrm{ABIC}=$ sample size adjusted BIC; LMR-LRT = Lo-Mendell-Rubin likelihood ratio test; BLRT = bootstrap likelihood ratio test.

$* p<.05$.

Low/Non-Smoking (35.24\%)

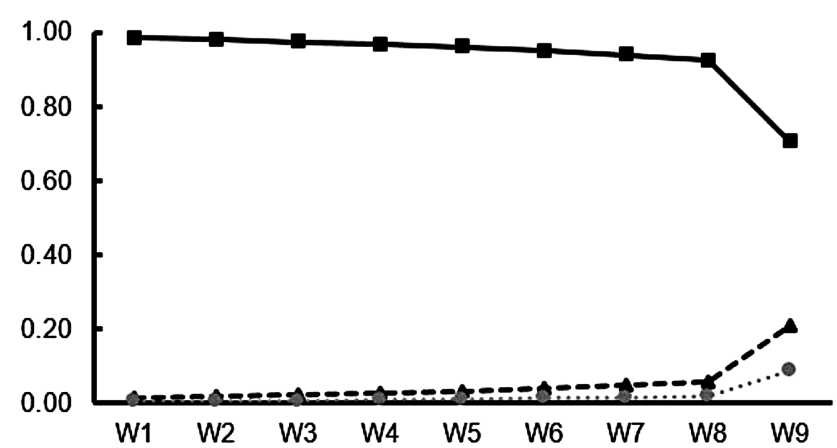

Mid-Adolescent Onset (21.63\%)

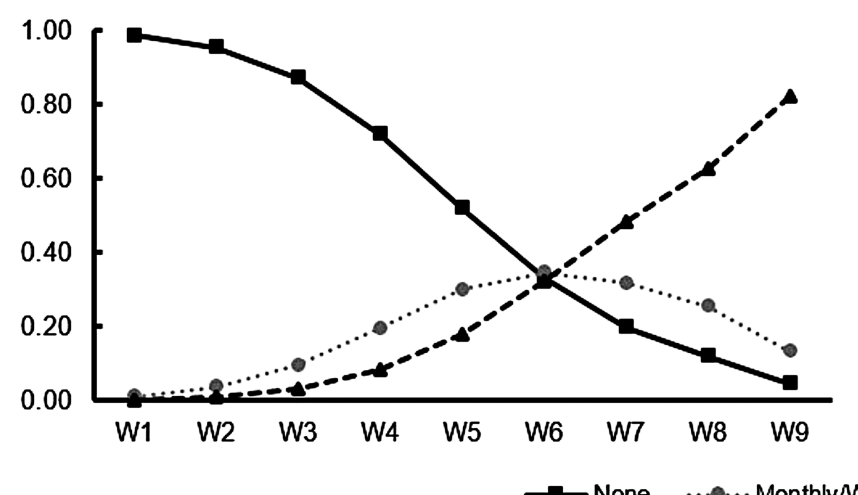

Occasional Smoking (17.21\%)

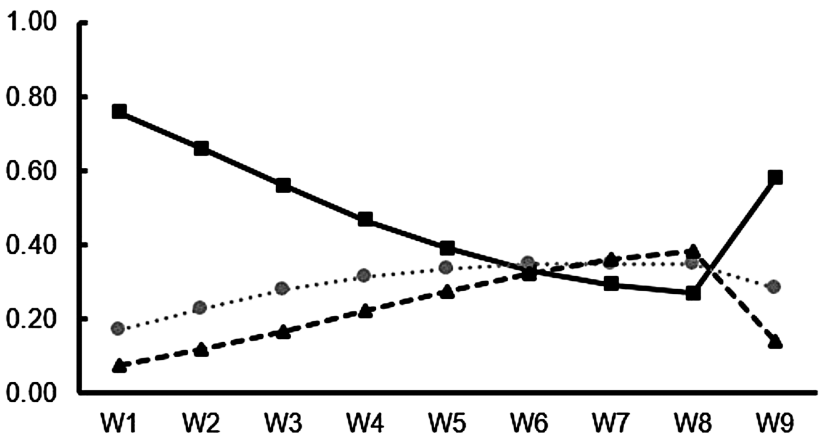

Early-Adolescent Onset (26.91\%)

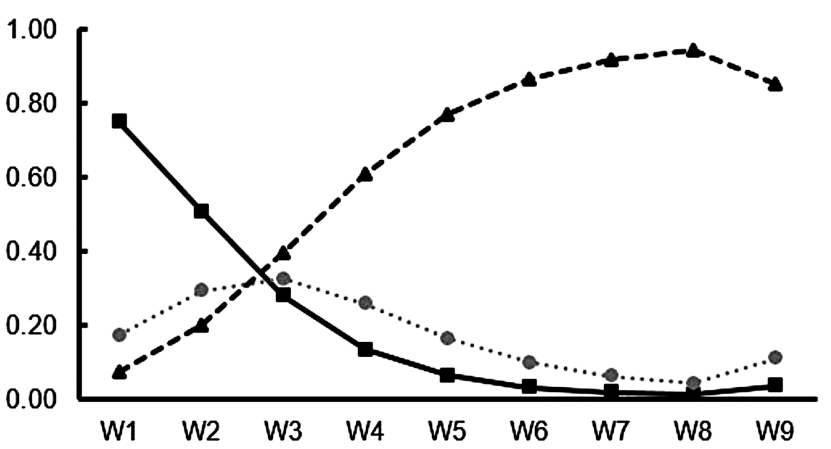

Figure 2. Probabilities ( $y$-axis) of smoking frequency by Wave ( $x$-axis) and latent trajectory class; W\# = Wave of Study; see also Supplementary Appendix A for exact probability values across classes.

Profiles of Smoking Latent Trajectory Classes

Table 2 presents descriptive statistics of the overall sample at baseline (Wave 1) and early adulthood (Wave 9), and differences in means/proportions across the four latent trajectory classes (see also Supplementary Appendixes B and C). We highlight only significant mean/proportion differences across the latent trajectory classes. Turning first to baseline demographics, relative to caretakers whose highest level of education is less than a high school diploma, the low/non-smoking group had higher proportions of participants whose caretaker's highest level of education is a college degree compared to the early-onset group. For gender, the occasional and early-onset groups had a higher proportion of females compared to the low/non-smoking group. Per capita family income means at baseline were significantly higher in the low/ non-smoking group compared to the other three groups.

Moving next to Wave 9 demographics, relative to those whose highest level of education is less than a high school diploma, a higher 
Table 2. Early Adolescent and Young Adult Demographic Profiles by Latent Smoking Trajectory Group ( $N=706)$

\begin{tabular}{|c|c|c|c|c|c|c|}
\hline & $\begin{array}{l}\text { Overall sample } \\
\qquad(N=706)\end{array}$ & $\begin{array}{l}\text { Non/low-probability } \\
\text { smokers }(N=255)\end{array}$ & $\begin{array}{c}\text { Occasional } \\
\text { smokers } \\
(N=110)\end{array}$ & $\begin{array}{l}\text { Mid-adolescent } \\
\text { onset }(N=151)\end{array}$ & $\begin{array}{l}\text { Early-onset } \\
(N=190)\end{array}$ & $F$-test \\
\hline \multicolumn{7}{|l|}{ Baseline demographics } \\
\hline Caretaker education & & & & & & $2.54, p=.01$ \\
\hline Less than high school (reference) & $13.69 \%$ & $11.37 \%$ & $18.18 \%$ & $14.57 \%$ & $13.51 \%$ & \\
\hline High school or GED & $34.00 \%$ & $27.06 \%{ }_{a}$ & $30.00 \%{ }_{a}$ & $35.76 \%_{a}$ & $44.22 \%_{\mathrm{a}}$ & \\
\hline Some college & $42.64 \%$ & $48.24 \% \%_{a}$ & $41.82 \%$ & $41.06 \%$ & $36.85 \%$ & \\
\hline College degree & $9.67 \%$ & $13.33 \% \%_{a}$ & $10.00 \%{ }_{a b}$ & $8.61 \%_{a b}$ & $5.42 \%_{b}$ & \\
\hline Female & $50.64 \%$ & $40.99 \%{ }_{a}$ & $59.09 \% \mathrm{o}_{\mathrm{b}}$ & $49.67 \% \%_{a}$ & $59.47 \%_{b}$ & $6.14, p=.00$ \\
\hline Resides on reservation & $86.05 \%$ & $83.73 \%_{a}$ & $88.98 \%{ }_{a}$ & $86.28 \% \%_{a}$ & $87.29 \%{ }_{a}$ & $0.55, p=.55$ \\
\hline $\begin{array}{l}\text { Per capita family income } \\
(\text { mean/SD })\end{array}$ & $5.70(4.98)$ & $7.04_{a}(6.14)$ & $5.16_{\mathrm{b}}(4.56)$ & $5.28_{b}(4.07)$ & $4.53_{b}(3.60)$ & $10.83, p=.00$ \\
\hline \multicolumn{7}{|l|}{ Current demographics } \\
\hline Resides on reservation & $65.90 \%$ & $60.87 \%$ a & $67.87 \% \%_{a}$ & $72.53 \%$ & $66.24 \% \%_{a}$ & $1.41, p=.24$ \\
\hline Current education & & & & & & $2.52, p=.01$ \\
\hline Less than high school (reference) & $27.89 \%$ & $20.24 \%$ & $30.11 \%$ & $31.48 \%$ & $34.03 \%$ & \\
\hline High school or GED & $39.24 \%$ & $36.44 \% \%_{a}$ & $35.04 \%{ }_{a}$ & $41.44 \% \%_{a}$ & $43.66 \%{ }_{a}$ & \\
\hline Some college & $21.00 \%$ & $23.91 \%_{a}$ & $24.11 \%_{\mathrm{ab}}$ & $18.93 \%{ }_{a b}$ & $16.94 \% \%_{b}$ & \\
\hline College degree & $11.88 \%$ & $19.42 \%_{\mathrm{a}}$ & $10.75 \%_{b}$ & $8.15 \%_{b}$ & $5.37_{\mathrm{b}}$ & \\
\hline Current parent & $66.38 \%$ & $55.33 \%$ & $66.71 \%_{\mathrm{ab}}$ & $70.46 \% \mathrm{bc}_{\mathrm{bc}}$ & $77.76 \%_{c}$ & $5.66, p=.00$ \\
\hline Individual income (mean/SD) & $19.55(20.24)$ & $23.34_{a}(22.13)$ & $20.68_{\mathrm{ab}}(17.19)$ & $16.25_{\mathrm{bc}}(16.29)$ & $16.45_{c}(17.61)$ & $6.45, p=.00$ \\
\hline \multicolumn{7}{|l|}{ Smoking characteristics } \\
\hline Lifetime nicotine dependence & $23.00 \%$ & $1.17 \% \%_{a}$ & $24.35 \%_{b}$ & $21.92 \%_{b}$ & $52.36 \%_{c}$ & $18.59, p=.00$ \\
\hline Caretaker smoking & $75.64 \%$ & $65.80 \% \%_{a}$ & $81.82 \% \mathrm{o}_{\mathrm{b}}$ & $77.10 \% \mathrm{o}_{\mathrm{b}}$ & $84.11 \%_{b}$ & $7.11, p=.00$ \\
\hline $\begin{array}{l}\text { Average number of quit attempts } \\
(\text { mean/SD })^{a}\end{array}$ & $2.22(4.77)$ & $2.08_{a}$ & $3.17_{\mathrm{a}}$ & $2.47_{a}$ & $1.80_{\mathrm{a}}$ & $10.45, p=.00$ \\
\hline $\begin{array}{l}\text { Average number cigarettes } \\
(\text { mean/SD })^{\mathrm{a}}\end{array}$ & $8.17(6.97)$ & $6.70_{\mathrm{ab}}$ & $5.63_{\mathrm{a}}$ & $8.83_{\mathrm{bc}}$ & $9.29_{c}$ & $63.09, p=.00$ \\
\hline
\end{tabular}

Proportion/means that do not share a subscript within rows are significantly different from one another $(p<.05)$; see Supplementary Appendixes B and C for full regression contrasts used to determine statistical differences between classes.

${ }^{a}$ Among current smokers (see also Supplementary Appendix C).

proportion of participants in the low/non-smoking group had a college degree compared to the other three groups. Moreover, relative to those whose highest level of education is less than a high school diploma, those in the low/non-smoking group has a higher proportion of participants whose highest level of education is some college compared to the early-onset group. The low/non-smoking group had the lowest probability of being a current parent compared to the mid- and early-onset groups. In addition, the occasional smoker group had a lower probability of being a current parent compared to the early-onset group. For current participant income, the means in the low/non-smoking group were significantly higher than the midand early-onset groups. The occasional smoking group also had a higher mean level of income compared to the mid- and early-onset groups.

Next, we examined groups vis-à-vis smoking characteristics. For cumulative lifetime nicotine dependence at Wave 8 (mean age: 18.3 years), the low/non-smoking group had fewer participants who met criteria compared to the other groups, whereas the early-onset group had the largest proportion of participants who met criteria for lifetime nicotine dependence compared to the other three groups. Occasional smokers and mid-onset smokers had statistically equivalent proportions of participants meeting nicotine dependence criteria. The low/non-smoking group had a lower proportion of participants whose caretakers smoked during adolescence compared to the other three groups. Among current smokers at Wave 9, there were no significant differences in the number of quit attempts made (the average among current smokers: 2.22, SD: 4.77). Current smokers in the low/non-smoking and occasional groups smoked fewer cigarettes per day than the early-onset group, and current smokers in the occasional smoking group smoked fewer cigarettes on average compared to the mid-onset group.

\section{Discussion}

Commercial cigarette use is highly prevalent among North American Indigenous people and is a key driver of health disparities. ${ }^{1,36}$ The overall early adulthood smoking rate found in the current study $(62 \%)$ is over four times higher than the national estimates of the same age group and higher than the national average for Indigenous adults as a whole. ${ }^{3,4}$ Of equal importance, smoking probabilities increased or remained stable from adolescence through the mid-to-late twenties, suggesting that smoking rates may continue to increase into early adulthood. Moreover, decades of research suggest that smokers make an average of 30 quit attempts before the successful cessation of 1 year or longer, ${ }^{37}$ which is nearly 10 times higher than the average number of quit attempts found in the current study. These findings align with prior research showing the high smoking prevalence and lower quit ratios among Indigenous populations, ${ }^{11}$ which run counter to broad public health targets (eg, Healthy People 2020, Canada's Tobacco Strategy). ${ }^{38,39}$ Taken together, the current study adds to a growing body of evidence signaling that antismoking public health initiatives have failed to reach Indigenous communities 
and/or are wholly inadequate. To reduce smoking-related health disparities, ${ }^{1,36}$ building effective public health policy requires a strong evidence base from which action can be taken. To fill this gap, we examined prospective latent trajectories and profiles of cigarette smoking frequency from adolescence to young adulthood among Indigenous people in the Upper Midwest of the United States and Canada.

Four latent trajectory classes emerged from the data, which is consistent with prior research. ${ }^{13,16,19,20}$ The composition of these latent trajectory groups, however, was less consistent. The largest single class consisted of low/non-smoking participants (35.2\%). Just under half $(48.5 \%)$ of the participants had high probabilities of being in classes characterized by escalation from low-probability smoking at various points in adolescence to current daily or near-daily smoking in young adulthood (early- and mid-adolescent onset). An additional $17.2 \%$ fit into a class with moderate, but declining rates of smoking (occasional smokers). Compared to prior smoking trajectory studies, the proportion of the sample in a low/non-smoking group was low. A majority of studies find that approximately half of participants fit into a similar trajectory group, ${ }^{13-15,17,20}$ whereas only about one-third of the participants in the current study were categorized into this group. With the exception of one study, ${ }^{23}$ the proportion of participants in the early-onset group was nearly double that found in other studies. ${ }^{13-15,17,20}$ Moreover, a substantial majority of participants who smoked were daily smokers, and fewer participants were considered experimental or light smokers compared to previous trajectory research. ${ }^{13,15,20}$

This analytic approach shows diverse patterns of use over the early life course, which can be used to identify critical periods of risk for onset, escalation, and desistance in use. The results overwhelmingly support the need for developmentally appropriate, early childhood smoking prevention programming. Efforts should aim to reduce the odds of transitioning from high rates of early experimental use to daily use patterns. A goal of national public health strategies is to reduce the proportion of smoking initiation cases among adolescents. ${ }^{38,39}$ Recent evidence suggests that some Indigenous groups are actually showing the increased prevalence and earlier onset smoking in the youngest age cohorts. ${ }^{7,8}$ The class characterized by early-adolescent onset use in this study had the highest probability of meeting lifetime nicotine dependence compared to the other four groups, and the mid-adolescent onset and occasional smoking groups had higher probabilities of lifetime nicotine dependence compared to the low/non-smoking group. Moreover, prior research suggests that smoking before the age of 16 and meeting criteria for nicotine dependence decreases the odds of long-term cessation..$^{22}$ As such, interventions aimed at delaying the onset of smoking may reduce the number of youth who start smoking and may increase the effectiveness of cessation efforts. In all but one group, smoking rates remained stable or increased into early adulthood, which suggests that delaying the onset of smoking would only be partially effective and interventions must persist across the life course.

The latent trajectory groups also varied by common demographic characteristics. At the individual level, females had the highest probability of early-onset smoking, whereas males had a higher probability of low/non-smoking or mid-adolescent onset smoking. Prior Indigenous research shows that females are more likely to initiate smoking at earlier ages and smoking into adulthood ${ }^{12}$ and have higher odds of nicotine dependence compared to males. ${ }^{25}$ Moreover, the results showed that low SES (eg, education and income) in childhood and young adulthood is associated with early onset and persistent patterns of cigarette use. These findings align with broader global trends in smoking, which document an enduring association between SES and cigarette smoking. ${ }^{24}$ At the family level, the results show that participants who grew up with caretakers who were smokers had a lower probability of being in the low/non-smoking group compared to the other three groups. Likewise, those who were categorized into the early- and mid-adolescent onset groups had a higher probability of being current parents compared to those in the low/non-smoking group. Taken together, these inequities require an intersectional, developmental approach to smoking policy, which can aid in identifying who is most at risk, the timing of this risk, and areas for focused public health interventions. This includes early gender-responsive programming, affordable access to cessation resources, and disrupting intergenerational cycles through comprehensive family-based interventions, including focused cessation efforts among caretakers and complete smoking bans in the home. ${ }^{10,40}$

Public health policy must also consider the unique local and cultural contexts of tribal communities. An important consideration for interpreting these findings is that traditional (ie, noncommercial) tobacco use has important cultural and spiritual meanings and has a different purpose, form, and function than commercial tobacco. ${ }^{41}$ The former poses no health risk when used traditionally, while the latter poses short- and long-term health risks. ${ }^{6}$ Enduring consequences of colonization and government sanctions on traditional spiritual practices have led to the conflation of traditional tobacco with modern commercial cigarettes. ${ }^{41}$ As a result, recreational, nontraditional tobacco use is considered normative, and the deleterious aspects of smoking are perceived to be of low importance in some Native communities. ${ }^{42,43}$ Tribally driven initiatives aim to change these norms and "keep tobacco scared." Moreover, federally recognized tribes are sovereign entities and have the capacity to potentiate community change through regulation and collective action (eg, tobacco taxation, smoking in public spaces). To reduce the community health burdens associated with commercial smoking, public health policy must be developed, implemented, and evaluated within the context of cultural respect and tribal sovereignty. ${ }^{41}$

\section{Limitations}

Several limitations warrant consideration. First, the data come from one Indigenous cultural group and may not be generalizable to others. Moreover, the initial sampling frame for the study included youth who resided on or near reservation/reserve land. As such, the results may not be generalizable to youth of the same cultural group who grew up in urban areas. Second, because we only had data on smoking quantity at the most recent wave of the study, smoking frequency was modeled instead of the more commonly used quantity $x$ frequency measures used in other studies. Prior research indicates that non-daily smokers are a heterogeneous group, and heavy nondaily smokers share similar smoking outcomes to daily smokers. Third, time ordering was not consistent across the profile indicators or for wave of the study. Nicotine dependence was last assessed at Wave 8 , which precludes a current assessment of nicotine dependence by a smoking group at Wave 9. Caretaker smoking was only assessed at Waves 5 and 7, but we suspect smoking rates among caretakers remained relatively stable over time. Relatedly, the gap in time between Waves 8 and 9 of the study was 7 years, which leaves a relevant developmental period with no data. We speculate that the inclusion of this data would not substantially alter the size or shape of the trajectories, but we might expect some additional context (eg, timing of escalation/desistance) for several of the groups. 
Despite these limitations, this study is unique in that it is the only within-culture study to examine prospective smoking trajectories across multiple relevant developmental periods. The results show a high smoking prevalence compared to national estimates. Smoking patterns are heterogeneous, unfold across the course of adolescence, and often persist through the transition to young adulthood. Likewise, these smoking trajectories can be clearly differentiated by demographic and smoking-related characteristics. These insights suggest multiple critical periods for smoking risk, and a general demographic risk profile, which can aid in developing targeted intervention and treatment programming in Indigenous communities.

\section{Supplementary Material}

A Contributorship Form detailing each author's specific involvement with this content, as well as any supplementary data, is available online at https://academic.oup.com/ntr.

\section{Funding}

This research was supported by the National Institute on Drug Abuse (R01DA13580, T32DA037183); National Institute of Mental Health (R01MH67281), and the National Institute on Alcohol Abuse and Alcoholism (R01AA020299). The content is solely the responsibility of the authors and does not necessarily represent the official views of the National Institutes of Health.

\section{Declaration of Interests}

The authors report no conflicts of interest

\section{Acknowledgments}

The authors would like to thank and acknowledge the Healing Pathways team, which includes David Bruyere, Laura Bruyere, Annabelle Jourdain, Priscilla Simard, Trisha Bruyere, Jake Becker, Laureen Bruyere, Frances Whitfield, GayeAnn Allen, Tina Handeland, Victoria Soulier, Bagwajikwe Madosh, Betty Jo Graveen, Clinton Isham, Carol Jenkins, Bill Butcher Jr., Delores Fairbanks, Devin Fineday, Bernadette Gotchie, Gloria Mellado. Christina Howard Marilyn Bowstring, Gary Charwood, Gina Stender, Roberta Roybal, Jim Bedeau, Kathy Dudley, Geraldine Brun, June Holstein, Frances Miller, Brenna Pemberton, Ed Strong, Barbara Thomas, Charity Prentice-Pemberton, FaLeisha Jourdain, Penny King, Valerie King, Linda Perkins, Christie Prentice, Gabe Henry, Howard Kabestra, Dallas Medicine, Glenn Cameron, Jackie Cameron, Gerilyn Fisher, Virginia Pateman, Irene Scott, Cindy McDougall, Whitney Accobee Celeste Cloud, Pat Moran, Stephanie Williams, Natalie Bergstrom, Bonnie Badboy, Elizabeth Kent, Sue Trnka, and Laurie Vilas.

\section{References}

1. Mowery PD, Dube SR, Thorne SL, Garrett BE, Homa DM, Nez Henderson P. Disparities in smoking-related mortality among American Indians/Alaska natives. Am J Prev Med. 2015;49(5):738-744.

2. Swaim RC, Stanley LR. Substance use among American Indian youths on reservations compared with a national sample of US adolescents. JAMA Netw Open. 2018;1(1):e180382.

3. Health Canada. Canadian Tobacco, Alcohol and Drugs Survey (CTADS): Summary of Results for 2017. Ottawa, ON: Health Canada.

4. Centers for Disease Control and Prevention. Current Cigarette Smoking among Adults in the United States. Atlanta, GA: Centers for Disease Control and Prevention.

5. Brave Heart MYH, Lewis-Fernández R, Beals J, et al. Psychiatric disorders and mental health treatment in American Indians and Alaska natives: results of the national epidemiologic survey on alcohol and related conditions. Soc Psychiatry Psychiatr Epidemiol. 2016;51(7):1033-1046.

6. Tanner JA, Henderson JA, Buchwald D, Howard BV, Henderson PN, Tyndale RF. Relationships between smoking behaviors and cotinine levels among two American Indian populations with distinct smoking patterns. Nicotine Tob Res. 2018;20(4):466-473.

7. Drope J, Liber AC, Cahn Z, et al. Who's still smoking? Disparities in adult cigarette smoking prevalence in the United States. CA Cancer J Clin. 2018;68(2):106-115.

8. Nez Henderson P, Kanekar S, Wen Y, et al. Patterns of cigarette smoking initiation in two culturally distinct American Indian tribes. Am J Public Health. 2009;99(11):2020-2025.

9. Patten CA, Koller KR, Flanagan CA, et al. Age of initiation of cigarette smoking and smokeless tobacco use among western Alaska Native people: secondary analysis of the WATCH study. Addict Behav Rep. 2019;9:100143.

10. Forster JL, Rhodes KL, Poupart J, Baker LO, Davey C. Patterns of tobacco use in a sample of American Indians in Minneapolis-St. Paul. Nicotine Tob Res. 2007;9 suppl (1):S29-S37.

11. Whitbeck LB, Armenta BE. Patterns of substance use initiation among Indigenous adolescents. Addict Behav. 2015;45:172-179.

12. Whitbeck LB, Hartshorn KJ, McQuillan J, Crawford DM. Factors associated with growth in daily smoking among indigenous adolescents. J Res Adolesc. 2012;22(4):768-781.

13. Audrain-McGovern J, Rodriguez D, Tercyak KP, Cuevas J, Rodgers K, Patterson F. Identifying and characterizing adolescent smoking trajectories. Cancer Epidemiol Biomarkers Prev. 2004;13(12):2023-2034.

14. Chassin L, Presson CC, Pitts SC, Sherman SJ. The natural history of cigarette smoking from adolescence to adulthood in a midwestern community sample: multiple trajectories and their psychosocial correlates. Health Psychol. 2000;19(3):223-231.

15. Hair E, Bennett M, Williams V, et al. Progression to established patterns of cigarette smoking among young adults. Drug Alcohol Depend. 2017;177(1):77-83.

16. Karp I, O'Loughlin J, Paradis G, Hanley J, Difranza J. Smoking trajectories of adolescent novice smokers in a longitudinal study of tobacco use. Ann Epidemiol. 2005;15(6):445-452.

17. Fuemmeler B, Lee CT, Ranby KW, et al. Individual- and communitylevel correlates of cigarette-smoking trajectories from age 13 to 32 in a U.S. population-based sample. Drug Alcohol Depend. 2013;132(1-2):301-308.

18. Mays D, Gilman SE, Rende R, Luta G, Tercyak KP, Niaura RS. Parental smoking exposure and adolescent smoking trajectories. Pediatrics. 2014;133(6):983-991.

19. Mathur C, Stigler MH, Erickson DJ, Perry CL, Forster JL. Transitions in smoking behavior during emerging adulthood: a longitudinal analysis of the effect of home smoking bans. Am J Public Health. 2014;104(4):715-720.

20. Riggs NR, Chou CP, Li C, Pentz MA. Adolescent to emerging adulthood smoking trajectories: when do smoking trajectories diverge, and do they predict early adulthood nicotine dependence? Nicotine Tob Res. 2007;9(11):1147-1154.

21. Colder CR, Mehta P, Balanda K, et al. Identifying trajectories of adolescent smoking: an application of latent growth mixture modeling. Health Psychol. 2001;20(2):127-135.

22. Breslau N, Peterson E. Smoking cessation in young adults: Age at initiation of cigarette smoking and other suspected influences. Am J Public Health. 1996;86(2):214-220.

23. White HR, Pandina RJ, Chen PH. Developmental trajectories of cigarette use from early adolescence into young adulthood. Drug Alcohol Depend. 2002;65(2):167-178.

24. Hiscock R, Bauld L, Amos A, Fidler JA, Munafò M. Socioeconomic status and smoking: a review. Ann N Y Acad Sci. 2012;1248(1):107-123.

25. Hautala D, Sittner K, Walls M. Onset, comorbidity, and predictors of nicotine, alcohol, and marijuana use disorders among North American Indigenous adolescents. J Abnorm Child Psychol. 2019;47(6):1025-1038. 
26. Whitesell NR, Asdigian NL, Kaufman CE, et al. Trajectories of substance use among young American Indian adolescents: patterns and predictors. $J$ Youth Adolesc. 2014;43(3):437-453.

27. Whitbeck L, Sittner Hartshorn K, Walls M. Indigenous Adolescent Development: Psychological, Social and Historical Contexts. New York, NY: Routledge; 2014.

28. Shaffer D, Fisher P, Lucas CP, Dulcan MK, Schwab-Stone ME. NIMH diagnostic interview schedule for children version IV (NIMH DISC-IV): description, differences from previous versions, and reliability of some common diagnoses. J Am Acad Child Adolesc Psychiatry. 2000;39(1):28-38.

29. Muthen B, Muthen L. Mplus. Los Angeles, CA: Muthen \& Muthen; 1998.

30. Lo Y, Mendell N, Rubin D. Testing the number of components in a normal mixture. Biometrika. 2001;88(3):767-778.

31. McLachlan G, Peel D. Finite Mixture Models. New York, NY: John Wiley \& Sons; 2000

32. StataCorp. Stata. College Station, TX: StataCorp; 2017.

33. White I, Royston P, Wood A. Multiple imputation using chained equations: issues and guidance for practice. Stat Med. 2011;30(4):377-399.

34. Jung T, Wickrama KAS. An introduction to latent class growth analysis and growth mixture modeling. Soc Personal Psychol Compass. 2008;2(1):302-317.

35. Muthen B. Latent variable analysis: growth mixture modeling and related techniques for longitudinal data. In: The SAGE Handbook of Quantitative Methodology for the Social Sciences. Thousand Oaks, CA: Sage Publications; 2004:345-368.
36. Espey DK, Jim MA, Cobb N, et al. Leading causes of death and all-cause mortality in American Indians and Alaska natives. Am J Public Health. 2014;104(S3):S303-S311.

37. Chaiton M, Diemert L, Cohen JE, et al. Estimating the number of quit attempts it takes to quit smoking successfully in a longitudinal cohort of smokers. BMJ Open. 2016;6(6):e011045.

38. U.S. Department of Health and Human Services, Office of Disease Prevention and Health Promotion. Healthy People 2020. Washington, DC: U.S. Department of Health and Human Services; 2019.

39. Health Canada. Canada's Tobacco Strategy. Ottawa, ON: Health Canada; 2019.

40. Kegler MC, Cleaver VL, Yazzie-Valencia M. An exploration of the influence of family on cigarette smoking among American Indian adolescents. Health Educ Res. 2000;15(5):547-557.

41. Boudreau G, Hernandez C, Hoffer D, et al. Why the world will never be tobacco-free: reframing "Tobacco Control" into a traditional tobacco movement. Am J Public Health. 2016;106(7):1188-1195.

42. Beauvais F, Jumper Thurman P, Burnside M, Plested B. Prevalence of American Indian adolescent tobacco use: 1993-2004. Subst Use Misuse. 2007;42(4):591-601.

43. Walls M, Hautala D, Gonzalez M, Greenfield B, Aronson B, Onella E. Perceptions and prevalence of alcohol and cigarette use among American Indian adults with type 2 diabetes. Clin Diabetes. $2019 ; 37(3): 260-269$. 